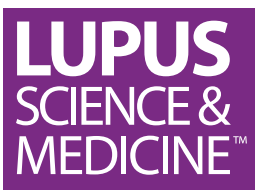

\title{
Safety, tolerability and pharmacokinetics of subcutaneous and intravenous anifrolumab in healthy volunteers
}

\author{
Raj Tummala, ${ }^{1}$ Tomas Rouse, ${ }^{2}$ Anna Berglind, ${ }^{2}$ Linda Santiago ${ }^{3}$
}

To cite: Tummala $\mathrm{R}$ Rouse T, Berglind A, et al. Safety, tolerability and pharmacokinetics of subcutaneous and intravenous anifrolumab in healthy volunteers. Lupus Science \& Medicine 2018;5:e000252. doi:10.1136/ lupus-2017-000252

Received 10 November 2017 Revised 5 February 2018 Accepted 15 February 2018
Check for updates

${ }^{1}$ AstraZeneca, Gaithersburg, Maryland, USA

${ }^{2}$ AstraZeneca, Gothenburg, Sweden

${ }^{3}$ Medlmmune, LLC, Mountain View, California, USA

Correspondence to

Dr Raj Tummala; Raj.Tummala@ astrazeneca.com

\begin{abstract}
Objectives To compare the pharmacokinetics (PK), safety and tolerability of subcutaneous (SC) and intravenous anifrolumab, an anti-type I interferon receptor monoclonal antibody in development for SLE, in healthy volunteers. Methods In this Phase I randomised, placebo-controlled study, 30 adults were assigned to three treatment cohorts (anifrolumab $300 \mathrm{mg} \mathrm{SC}(\mathrm{n}=6)$, anifrolumab $300 \mathrm{mg}$ intravenous ( $\mathrm{n}=6)$, anifrolumab $600 \mathrm{mg} \mathrm{SC}(\mathrm{n}=6))$ and placebo ( $n=4 /$ cohort). Serial blood samples were collected up to Day 84 to measure anifrolumab concentrations and antidrug antibodies (ADAs). PK parameters were estimated by noncompartmental analysis.

Results Maximum serum concentrations in SC cohorts occurred after 4-7 days. Anifrolumab serum concentrations were below the limit of detection in all individuals by Day 84. Exposure to SC anifrolumab increased dose proportionally from $300 \mathrm{mg}$ to $600 \mathrm{mg}$ based on area under the serum concentration-time curve. Anifrolumab $300 \mathrm{mg}$ SC exposure reached $87 \%$ of the intravenous exposure. Anifrolumab $300 \mathrm{mg} \mathrm{SC}$ and placebo administration elicited minimal injection-site reactions. Transient injection-site induration occurred in five of six individuals after anifrolumab $600 \mathrm{mg} \mathrm{SC}$ and two of four individuals after placebo. Transient, mild to moderate injection-site induration and pruritus occurred simultaneously in two of six individuals after anifrolumab $600 \mathrm{mg} \mathrm{SC}$. Adverse events were reported by $50 \%(n=9)$ of anifrolumabtreated individuals and $33 \%(n=4)$ of placebo-treated individuals. ADAs were detected in only one individual in the anifrolumab 300-mg intravenous group at the Day 84 assessment.

Conclusion Anifrolumab 300-mg SC exposure was $87 \%$ of intravenous administration, with single SC anifrolumab administrations well tolerated in healthy volunteers.
\end{abstract}

\section{INTRODUCTION}

SLE is a chronic, multisystem autoimmune disease with extreme heterogeneity between patients with respect to clinical manifestations, organ involvement and disease severity. ${ }^{1}$ The incidence and prevalence of SLE are estimated to be as great as 32 cases per 100000 individuals per year and 520 cases per 100000 individuals, respectively. ${ }^{1}$ Although the 5 -year survival rate has increased to almost $90 \%$ in the last 70 years, there remains an unmet need for therapeutics that achieve stable low disease activity, prevent ongoing organ damage and reduce the need for high-dosage corticosteroid use. $^{2}$

Increased clarity of the pathogenesis of SLE is driving development of immunotherapy targeted to specific patient endotypes. A hallmark of SLE is the presence of self-reactive autoantibodies bound to circulating nuclear antigens, which drive cellular overproduction of type I interferon (IFN) and other proinflammatory cytokines. ${ }^{3}$ A substantial body of evidence supports an important role of type I IFNs in the pathogenesis of SLE. ${ }^{4-9}$ The successful development of anti-type I IFN therapeutics is anticipated to advance the medical management of patients with SLE significantly.

Anifrolumab, a fully human immunoglobulin $\mathrm{G}_{1}\left(\mathrm{IgG}_{1}\right)$ monoclonal antibody targeting type I IFN alpha receptor 1 (IFNAR1), is in Phase III development as an intravenous therapeutic for moderate to severe SLE. Anifrolumabinhibits type IIFN-dependent cell signalling by binding to IFNAR1 and blocking formation of the IFN/IFNAR complex..$^{10}$ In the Phase IIb MUSE trial (NCT01438489), anifrolumab $300 \mathrm{mg}$ every 4 weeks intravenously plus standard of care significantly decreased SLE disease activity across a range of endpoints, especially for patients with type I IFN gene signature test high results at baseline. $^{7}$ Two Phase III trials (TULIP-I/II; NCT02446899/NCT02446912) are currently ongoing to further evaluate the efficacy and safety of intravenous anifrolumab for patients with moderate to severe SLE.

Of the three novel anti-type I IFN antibodies (rontalizumab, sifalimumab and anifrolumab) evaluated in Phase II clinical trials for SLE, the intravenous formulation of anifrolumab had the highest 


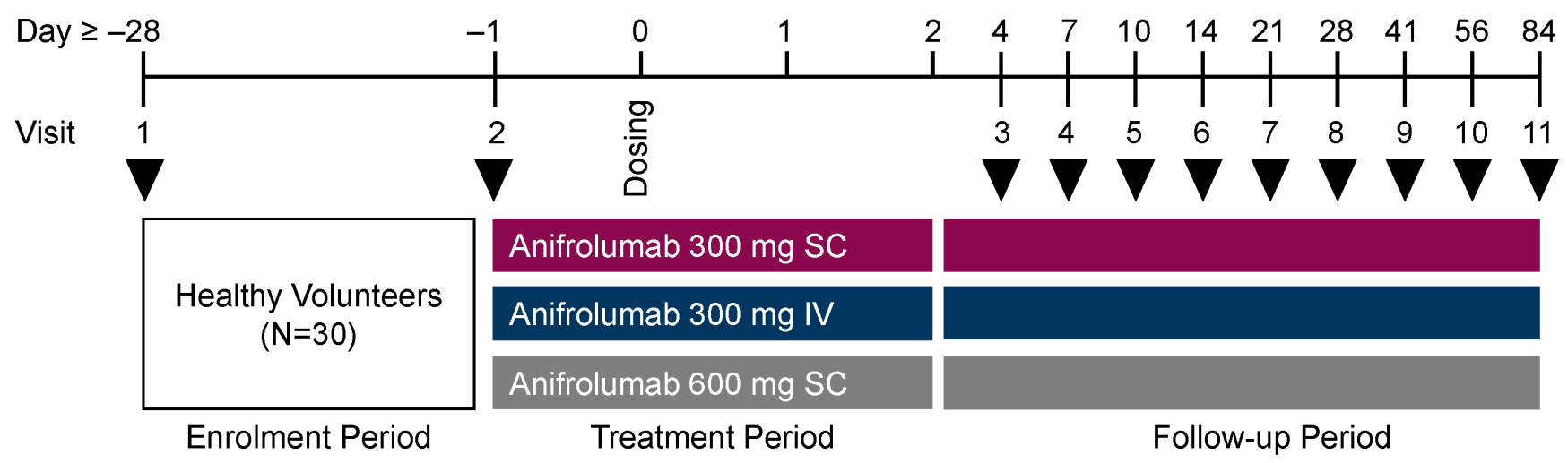

Figure 1 Study design. IV, intravenous; SC, subcutaneous. Black arrow heads refer to visits.

efficacy relative to placebo. ${ }^{711} 12$ However, patients and health care providers generally prefer subcutaneous (SC) over intravenous administration of biologics. ${ }^{13-18}$ Thus, we profiled the pharmacokinetics (PK), safety and tolerability of anifrolumab administered SC and intravenously to healthy volunteers.

\section{PATIENTS AND METHODS \\ Population, study design and objectives}

This was a Phase I, single-centre (Baltimore, Maryland, USA), double-blind, randomised, placebo-controlled trial (NCT02601625) involving healthy volunteers. The trial included individuals aged $18-55$ years with a body mass index of $18-32 \mathrm{~kg} / \mathrm{m}^{2}$ and body weight $\geq 50 \mathrm{~kg}$. Additional inclusion criteria comprised a normal Pap smear for female volunteers, sufficient abdominal adipose tissue for SC injection, no prior or current latent or active tuberculosis and no history of recent or severe Herpes zoster infection. Exclusion criteria included any recent infection requiring hospitalisation or treatment with parenteral antimicrobials, any severe herpes infection at any time prior to dosing and receipt of a recent live or attenuated vaccine administration.

The study consisted of an enrolment and screening period of up to 28 days, a 3-day residential period and follow-up visits on Days 4, 7, 10, 14, 21, 28, 42, 56 and 84 (figure 1). Volunteers were assigned to three sequential treatment cohorts of equal size and randomised within each cohort to receive a single dose of either anifrolumab ( $\mathrm{n}=6$ / cohort) or placebo $(0.9 \%$ normal saline; $\mathrm{n}=4 /$ cohort $)$. For Cohort $1(\mathrm{n}=10)$, anifrolumab $300 \mathrm{mg}$ or placebo was administered as two separate 1-mL SC injections via syringe and 27-gauge $1 / 2$ " needle. For Cohort $2(\mathrm{n}=10)$, anifrolumab $300 \mathrm{mg}$ or placebo was administered as a $100-\mathrm{mL}$ intravenous infusion over $30 \mathrm{~min}$. For Cohort $3(\mathrm{n}=10)$, anifrolumab $600 \mathrm{mg}$ or placebo was administered as $4 \mathrm{~mL}$ SC by large-volume infusion pump (Perfusor Space Infusion Pump, B. Braun Medical, Inc.). SC injectates were administered in the anterior abdominal wall while avoiding a 5 -cm radius around the umbilicus.
Pharmacokinetics, safety, tolerability and immunogenicity The objective of this study was to evaluate the PK, safety, tolerability and immunogenicity of single SC and intravenous administrations of anifrolumab. Serial venous blood samples were collected pre dose, $5 \mathrm{~min}$ after SC injection or intravenous infusion ended, 24 and 48 hours post dose and at Visits 3-11. Samples were analysed to measure serum anifrolumab concentrations with a validated assay, as previously described. ${ }^{19}$ Serum from blood samples collected pre dose and at Visits 3, 8 and 11 was evaluated also for the presence of anti-drug antibodies using a validated bioanalytical method. ${ }^{19}$

Safety endpoints included adverse events, adverse events of special interest, laboratory assessments and vital signs. Adverse events of special interest were serious infections, opportunistic infections, anaphylaxis, malignancy, Herpes zoster, tuberculosis (including latent tuberculosis), influenza, vasculitis and major adverse cardiac events (including stroke, myocardial infarction, or cardiovascular death).

Tolerability endpoints included assessments of the SC injection sites for pain (100-mm visual analogue scale (VAS)), pruritus (100-mm VAS), erythema (largest diameter, $\mathrm{mm}$ ) and induration (largest diameter, $\mathrm{mm}$ ). Injection-site assessments occurred at 10, 20 and $30 \mathrm{~min}$ and then 1, 2, 4, 8, 24 and 48 hours post injection. Injection-site reactions were not recorded as adverse events unless an individual complained of these at any time point post injection.

\section{Statistical analysis}

The safety analysis set included all individuals who received at least one dose of anifrolumab or placebo. The PK analysis set included all individuals in the safety analysis set for whom at least one primary PK parameter could be calculated. Data were summarised by descriptive statistics. PK parameters were calculated by non-compartmental analysis with Phoenix WinNonlin V/6.2 (Certara, Inc., Princeton, New Jersey, USA) and included the area under the serum concentration-time curve (AUC), clearance $(\mathrm{CL}, \mathrm{CL} / \mathrm{F})$, maximum serum concentration $\left(\mathrm{C}_{\max }\right)$ and time to reach maximum serum concentration $\left(\mathrm{t}_{\max }\right)$. All 
Table 1 Demographics and baseline characteristics

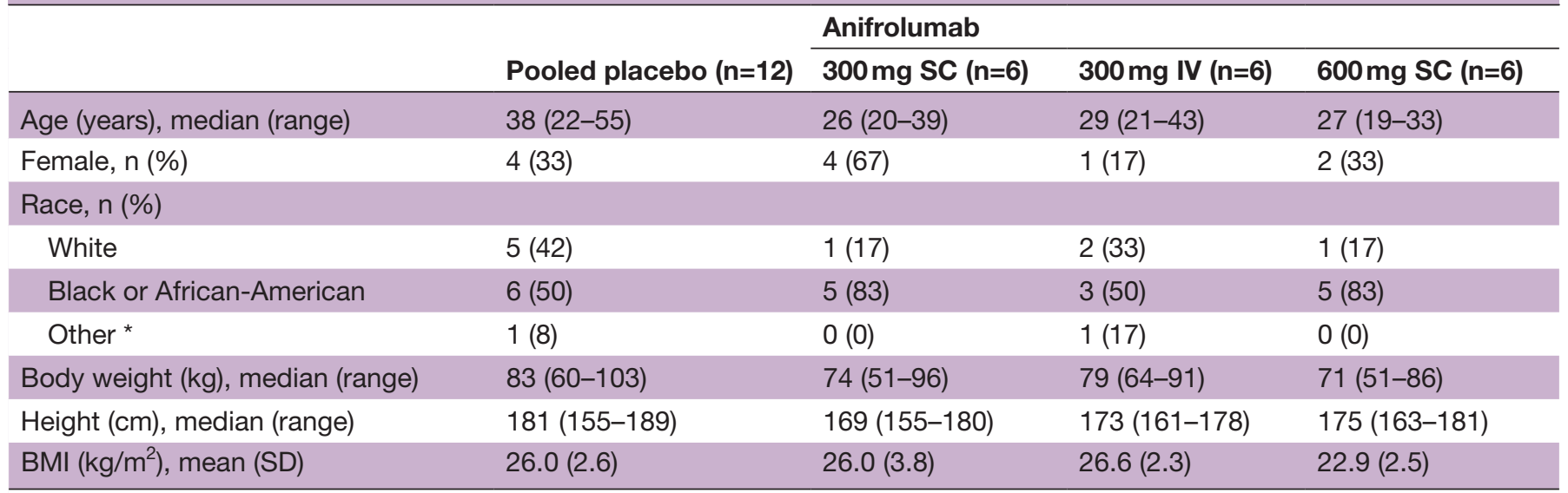

${ }^{*}$ Native Hawaiian or other Pacific Islander or Asian.

BMI, body mass index; IV, intravenous; SC, subcutaneous.

data were analysed with SAS System V.9.2 (SAS Institute, Inc., Cary, NC, USA) .

\section{RESULTS}

Volunteer disposition

Thirty adult healthy volunteers were randomised to and completed treatment, and $28(93 \%)$ individuals completed the study (table 1). Individuals had a median age of 30 years (range: 19-55 years), and a mean body mass index of $25.4 \mathrm{~kg} / \mathrm{m}^{2}$ (SD: $3.0 \mathrm{~kg} / \mathrm{m}^{2}$ ). Most individuals were black or African-American (63\%), and more than half of the volunteers were male $(63 \%)$. Two individuals were lost to follow up: one who received placebo in the anifrolumab 600-mg group after Visit 6 (Day 14) and one who received anifrolumab in the anifrolumab 600-mg group after Visit 7 (Day 21). All 30 individuals were included in the PK and safety analyses sets.

\section{Anifrolumab pharmacokinetics}

In the SC cohorts, $\mathrm{C}_{\max }$ (mean (SD)) was 36.2 (11.6) $\mu \mathrm{g} / \mathrm{mL}$ in the anifrolumab 300-mg group and 63.9 (20.5) $\mu \mathrm{g} / \mathrm{mL}$ in the anifrolumab 600-mg group. Peak serum concentrations $\left(\mathrm{T}_{\max }\right)$ occurred 4-7 days after injection (table 2 and figure 2). Exposure to SC anifrolumab increased approximately dose proportionally from $300 \mathrm{mg}$ to $600 \mathrm{mg}$ based on mean (SD) AUC (anifrolumab $300 \mathrm{mg}$ : 785 (331) day $\cdot \mu \mathrm{g} / \mathrm{mL}$; anifrolumab $600 \mathrm{mg}: 1828$ (680) day $\cdot \mu \mathrm{g} / \mathrm{mL}$; table 2). At the 300-mg dose, anifrolumab exposure after SC administration reached approximately $87 \%$ of the intravenous administration exposure (mean AUC (SD): 907 (175) day. $\mu \mathrm{g} / \mathrm{mL}$ ). All individuals had quantifiable serum anifrolumab concentrations in all samples from the time of dosing until at least 28 days post dose. Anifrolumab serum concentrations were below the limit of detection for all individuals by 84 days post dose (figure 2).

\section{Safety, tolerability and immunogenicity}

Adverse events were reported by $50 \% \quad(n=9)$ and $33 \%$ $(n=4)$ of anifrolumab-treated and placebo-treated individuals, respectively (table 3 ). The most common adverse events in anifrolumab-treated individuals were upper respiratory tract infection $(\mathrm{n}=3 ; 17 \%)$ and dry throat $(\mathrm{n}=2 ; 11 \%)$. No serious adverse events were reported (table 3). Anti-drug antibodies were detected in only one individual in the anifrolumab 300-mg intravenous group at the Day 84 assessment.

Injection-site pain was either absent (600-mg cohort) or minimal to mild (300-mg cohort) and resolved within

Table 2 Anifrolumab serum PK parameters following subcutaneous and intravenous administration of anifrolumab

\begin{tabular}{|c|c|c|c|}
\hline & \multicolumn{3}{|l|}{ Anifrolumab } \\
\hline & $300 \mathrm{mg}$ SC $(n=6)$ & $300 \mathrm{mg}$ IV $(n=6)$ & $600 \mathrm{mg} \mathrm{SC}(\mathrm{n}=6)^{*}$ \\
\hline AUC (day· $\mu \mathrm{g} / \mathrm{mL})$, mean $(\mathrm{SD})$ & $785(331)$ & $907(175)$ & $1828(680)$ \\
\hline$t_{\max }$ (day), median (range) & $4.1(4.0-7.0)$ & $0.03(0.03-1.03)$ & $7.0(4.0-9.0)$ \\
\hline CL (L/day), mean (SD) & NA & $0.34(0.05)$ & NA \\
\hline
\end{tabular}

${ }^{*} \mathrm{~N}=5$ for $\mathrm{AUC}$ and $\mathrm{CL} / \mathrm{F}$ because one individual was lost to follow-up after Day 22.

AUC, area under the serum concentration-time curve from time 0 extrapolated to infinity; $\mathrm{C}_{\max }$, maximum serum concentration; $\mathrm{CL}$, total body clearance after intravenous administration; CL/F, apparent total body clearance after extravascular administration estimated as dose divided by AUC; IV, intravenous; NA, not applicable; SC, subcutaneous; $\mathrm{t}_{\max }$, time to reach maximum serum concentration. 


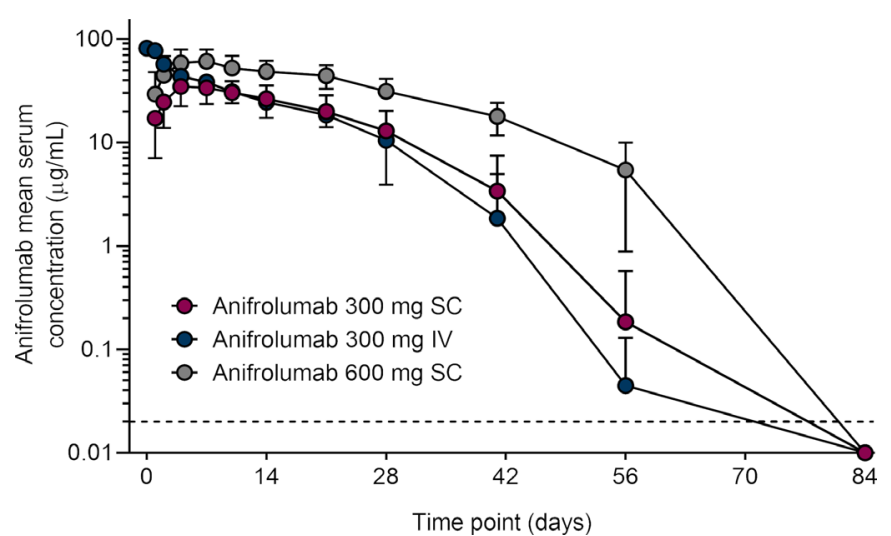

Figure 2 Mean serum concentration time profiles of anifrolumab following subcutaneous and intravenous administration of anifrolumab. ${ }^{a}$ a Data below the limits of detection are plotted as one-half of the lower limits of quantification $(0.02 \mu \mathrm{g} / \mathrm{mL}$; dashed line). Values are means and SD. IV, intravenous; SC, subcutaneous.

10 min after administration (figure $3 \mathrm{~A}$ ). Injection-site pruritus was minimal after administering anifrolumab or placebo in the 300-mg SC cohort and was mild to moderate in two individuals who received anifrolumab $600 \mathrm{mg}$ (figure 3B). Pruritus resolved by $20 \mathrm{~min}$ post dose in both cohorts. Injection-site erythema immediately after SC dosing was more pronounced in individuals receiving the larger volume infusion (600-mg cohort) compared with those receiving the SC injection (300-mg cohort; figure $3 \mathrm{C}$ ). There were minimal differences in erythema size between anifrolumab and placebo groups in the 600-mg cohort. Erythema resolved within 8 hours post dose in all cohorts. Injection-site induration was more pronounced immediately after $600-\mathrm{mg}$ SC infusions than after 300-mg SC injections (figure 3D). Induration resolved in all groups by 4 hours post dose. Induration was not associated with pain or erythema.

\section{DISCUSSION}

Unresolved medical needs, such as insufficient disease control and toxicity of current therapies, and a better understanding of the pathobiology of lupus have stimulated development of endo-type-specific therapeutics over the past decade. More than 25 novel agents are undergoing Phase II/III clinical trials for the treatment of SLE (https://clinicaltrials.gov/). Antibodies targeting the type I IFN pathway, rontalizumab, sifalimumab and anifrolumab, have recently been assessed in Phase II randomised controlled trials. ${ }^{711} 12$ Primary endpoints were not met in the rontalizumab trial but were reached in the sifalimumab trial. More patients (58.3\%) receiving 200-mg sifalimumab intravenously achieved an SLE Responder Index of 4 points (SRI (4)) at Day 365 compared with those receiving placebo $(45.4 \%)$. The anifrolumab trial also reached its primary endpoint of an SRI(4) and sustained oral corticosteroid reduction at Day 169. This composite endpoint was achieved by $34.3 \%$ of patients in the 300-mg anifrolumab intravenous group compared with $17.6 \%$ of patients receiving placebo. A similar difference in SRI(4) was observed at Day 365 (300-mg anifrolumab intravenous: $51.5 \%$ vs placebo: $25.5 \%$ ). Although these antibodies have not been compared side by side in a single trial, the data suggest that intravenous anifrolumab has best-in-disease efficacy for this class of drug in patients with moderate to severe SLE. ${ }^{20}$ The intravenous formulation of anifrolumab is undergoing further evaluation in Phase III trials (NCT02446899 and NCT02446912).

In the past 50 years, only one new therapeutic, belimumab, has been approved for the treatment of SLE. The approval of intravenous anifrolumab would be a significant advancement in the management of SLE, and the potential for SC administration would be further advantageous because patients and health care providers prefer SC over intravenous injections. ${ }^{17}$ Several studies have evaluated patients' preference after receiving biologicals via both intravenous and SC routes of administration. ${ }^{1314161821}$ Patients cited time saving, convenience and avoidance of venipuncture as the primary reasons they preferred the SC route of administration. The presence of transient, mild to moderate injection-site reactions after SC, but not intravenous, administration of a biologic was not a sufficient deterrent.

Notwithstanding the evidence that patient preference and pharmacoeconomics are the primary drivers of SC formulation development, initial studies are required to demonstrate that the SC route of administration has comparable drug exposure, safety, efficacy and tolerability to an intravenous formulation. ${ }^{22}$ Accordingly, we evaluated the PK, safety and tolerability of SC and

Table 3 Number of volunteers who had at least one adverse event

\begin{tabular}{|c|c|c|c|c|}
\hline & \multirow[b]{2}{*}{ Pooled placebo $(n=12)$} & \multicolumn{3}{|l|}{ Anifrolumab } \\
\hline & & $300 \mathrm{mg} \mathrm{SC}(\mathrm{n}=6)$ & $300 \mathrm{mg}$ IV $(n=6)$ & $600 \mathrm{mg} \mathrm{SC}(n=6)$ \\
\hline Any AE, n (\%) & $4(33)$ & $3(50)$ & $2(33)$ & $4(67)$ \\
\hline Any AE (with an outcome of death), n (\%) & $0(0)$ & $0(0)$ & $0(0)$ & $0(0)$ \\
\hline Any serious $\mathrm{AE}^{*}, \mathrm{n}(\%)$ & $0(0)$ & $0(0)$ & $0(0)$ & $0(0)$ \\
\hline $\begin{array}{l}\text { Any AE causing discontinuation } \\
\text { of product, } n(\%)\end{array}$ & $0(0)$ & $0(0)$ & $0(0)$ & $0(0)$ \\
\hline
\end{tabular}

*Includes events with an outcome of death.

AE, adverse event; IV, intravenous; SC, subcutaneous. 


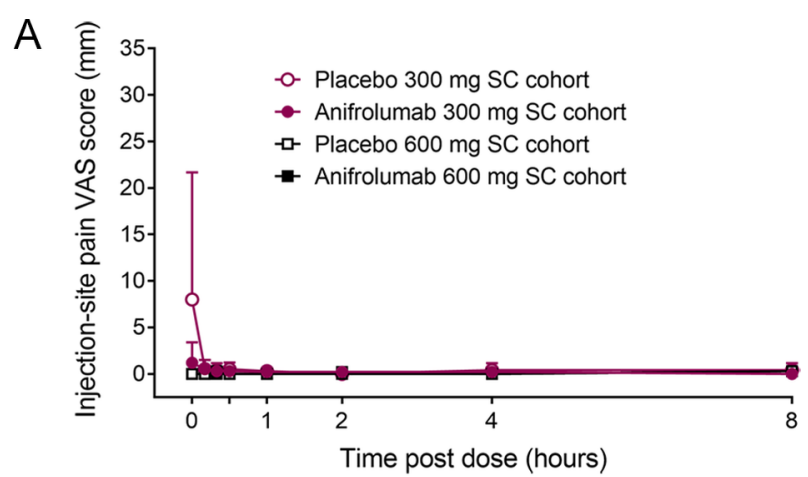

$\mathrm{B}$

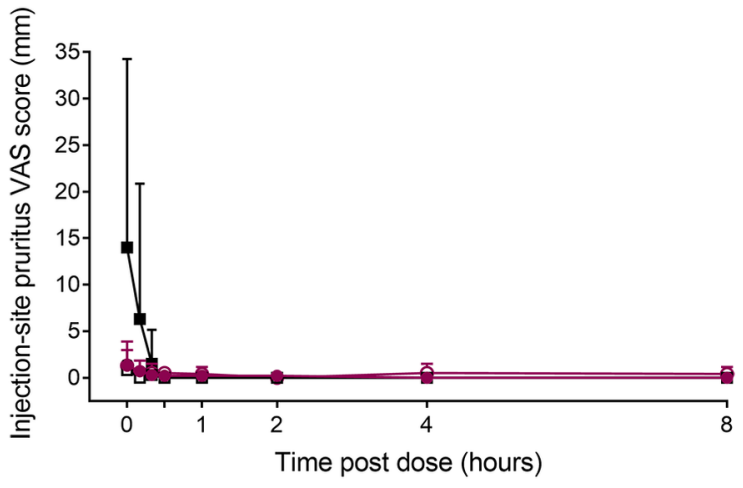

C

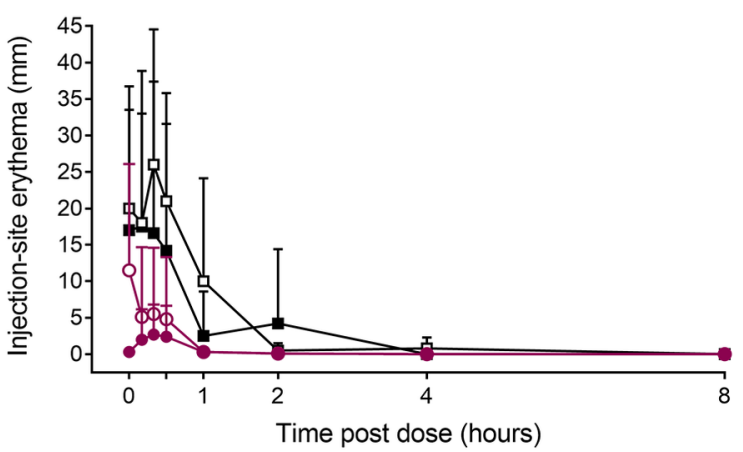

D

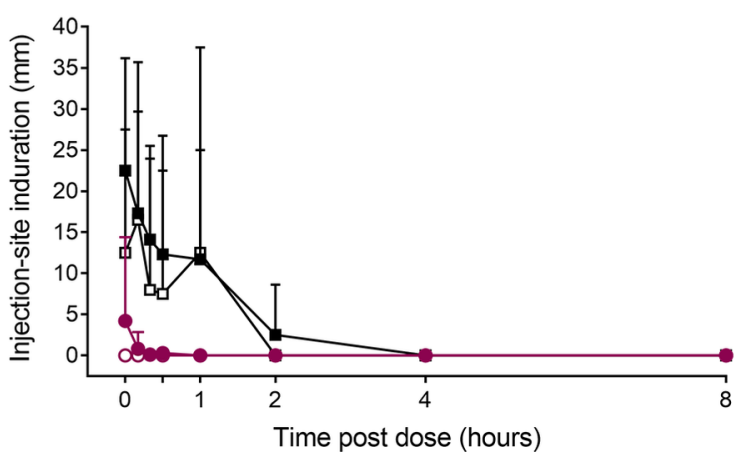

Figure 3 Injection-site reactions after subcutaneous administration of anifrolumab and placebo. Values are means and SD. SC, subcutaneous; VAS, visual analogue scale.

intravenous administration of anifrolumab for healthy volunteers. We found that the exposure of anifrolumab $300 \mathrm{mg}$ SC was approximately $87 \%$ of intravenous administration and that SC anifrolumab was well-tolerated and with minimal injection-site reactions. In our study, the incidence and magnitude of these reactions were consistent with prior studies. ${ }^{1314161821}$
In general, the pharmacokinetic properties of monoclonal antibodies are markedly different to most small molecule drugs. ${ }^{23}$ The larger molecular size of antibodies contributes to slower absorption from SC injection sites via the lymphatics, slower distribution to tissues and lower volumes of distribution. If there is significant presystemic catabolism of the subcutaneously administered antibody, bioavailability is often low to intermediate. ${ }^{24}$ The times to peak serum concentration and bioavailabilities of registered $\mathrm{IgG}_{1}$ monoclonal antibodies marketed as $\mathrm{SC}$ formulations typically range from 3 to 8 days and $60 \%-80 \%$, respectively. ${ }^{25}$ The $\mathrm{t}_{\max }$ for anifrolumab after $\mathrm{SC}$ administration is consistent with this range. In contrast, anifrolumab exposure after SC administration suggests that the bioavailability of the antibody may be relatively high. Collectively, our data support the further development of the SC formulation of anifrolumab. The pharmacokinetics, pharmacodynamics and safety of multiple SC administrations of anifrolumab are currently being evaluated in a Phase II trial (NCT02962960). This placebo-controlled study is assessing two fixed dosages of anifrolumab $(150 \mathrm{mg}$ and $300 \mathrm{mg}$ ) administered subcutaneously every 2 weeks for up to 50 weeks, within a treatment period of 52 weeks. The objective of the study is to identify the most appropriate SC dosage for a future Phase III trial.

A limitation of our study was the absence of pharmacodynamic assessments after SC and intravenous administration of anifrolumab to healthy volunteers. The primary pharmacodynamic effect of anifrolumab in patients with SLE is suppression of the type I IFN gene signature. Increased type I IFN expression initiates multiple signal transduction pathways, which in turn lead to expression of up to 2000 IFN-stimulated genes. ${ }^{26}$ Anifrolumab $300 \mathrm{mg}$ intravenously administered to patients with moderate to severe SLE every 4 weeks for 48 weeks suppressed the type I IFN gene signature with a median neutralisation range of $85 \%-90 \%$ from Day 29 to Day $365 .{ }^{27}$ The IFN gene signature is relatively low in healthy volunteers, precluding assessments of anifrolumab pharmacodynamics in our study. ${ }^{28}$ Additional limitations were that the volunteers were mostly black or African-American and male. The pharmacodynamics of SC anifrolumab will be assessed in patients with SLE during the Phase II clinical trial described earlier.

In conclusion, SC administration of anifrolumab $300 \mathrm{mg}$ and anifrolumab $600 \mathrm{mg}$ exhibited dose-proportional pharmacokinetics in healthy volunteers. Anifrolumab exposure after SC administration was relatively high compared with similar studies of registered $\mathrm{IgG}_{1}$ monoclonal antibodies marketed as $\mathrm{SC}$ formulations. Anifrolumab, administered as an intravenous infusion or an SC injection, was well tolerated in healthy subjects. Collectively, these data support the further development of anifrolumab as an SC formulation for the treatment of patients with lupus disorders.

Acknowledgements Some of these data have been published in abstract form following presentation at the 2017 Annual Meeting of the American College of Rheumatology (Tummala R, Rouse T, Berglind A, Santiago L. Arthritis Rheumatol. 
2017; 69 (suppl 10)). Editorial support was provided by Francis J Golder, BVSc, PhD, of JK Associates, Inc., and Michael A Nissen, ELS, of AstraZeneca. This support was funded by AstraZeneca.

Contributors RT proposed the study design. LS performed the pharmacokinetics analysis. TR and $A B$ performed the statistical analyses. All authors contributed to developing the manuscript.

Funding This study was funded by AstraZeneca.

Competing interests RT, TR and $A B$ are employees of AstraZeneca. LS is an employee of Medlmmune LLC and a shareholder of AstraZeneca.

Patient consent All volunteers provided informed consent before the start of the study.

Ethics approval The clinical protocol was approved by the institutional review board and independent ethics committee.

Provenance and peer review Not commissioned; externally peer reviewed. Data sharing statement The study protocol is available upon request. Study data are available upon request and completion of a data sharing agreement with AstraZeneca via the Data Request Portal (https://astrazenecagroup-dt.pharmacm. com//DT/Home/Index/).

Open Access This is an Open Access article distributed in accordance with the Creative Commons Attribution Non Commercial (CC BY-NC 4.0) license, which permits others to distribute, remix, adapt, build upon this work non-commercially, and license their derivative works on different terms, provided the original work is properly cited and the use is non-commercial. See: http://creativecommons.org/ licenses/by-nc/4.0/

(c) Article author(s) (or their employer(s) unless otherwise stated in the text of the article) 2018. All rights reserved. No commercial use is permitted unless otherwise expressly granted.

\section{REFERENCES}

1. Carter EE, Barr SG, Clarke AE. The global burden of SLE: prevalence, health disparities and socioeconomic impact. Nat Rev Rheumatol 2016;12:605-20.

2. Lateef $A$, Petri M. Unmet medical needs in systemic lupus erythematosus. Arthritis Res Ther 2012;14(Suppl 4):S4.

3. Tsokos GC, Lo MS, Costa Reis P, et al. New insights into the immunopathogenesis of systemic lupus erythematosus. Nat Rev Rheumatol 2016;12:716-30.

4. Deng Y, Tsao BP. Advances in lupus genetics and epigenetics. Curr Opin Rheumatol 2014;26:482-92.

5. Weckerle CE, Franek BS, Kelly JA, et al. Network analysis of associations between serum interferon- $\alpha$ activity, autoantibodies, and clinical features in systemic lupus erythematosus. Arthritis Rheum 2011;63:1044-53.

6. Dall'era MC, Cardarelli PM, Preston BT, et al. Type I interferon correlates with serological and clinical manifestations of SLE. Ann Rheum Dis 2005;64:1692-7.

7. Furie R, Khamashta M, Merrill JT, et al. Anifrolumab, an antiInterferon- $\alpha$ receptor monoclonal antibody, in moderate-to-severe systemic lupus erythematosus. Arthritis Rheumatol 2017;69:376-86.

8. Agrawal H, Jacob N, Carreras E, et al. Deficiency of type I IFN receptor in lupus-prone New Zealand mixed 2328 mice decreases dendritic cell numbers and activation and protects from disease. $J$ Immunol 2009;183:6021-9.

9. Liu Z, Bethunaickan R, Huang W, et al. Interferon- $\alpha$ accelerates murine systemic lupus erythematosus in a T cell-dependent manner. Arthritis Rheum 2011;63:219-29.
10. Peng L, Oganesyan $\mathrm{V}, \mathrm{Wu} \mathrm{H}$, et al. Molecular basis for antagonistic activity of anifrolumab, an anti-interferon- $\alpha$ receptor 1 antibody. MAbs 2015;7:428-39.

11. Khamashta M, Merrill JT, Werth VP, et al. Sifalimumab, an antiinterferon- $\alpha$ monoclonal antibody, in moderate to severe systemic lupus erythematosus: a randomised, double-blind, placebocontrolled study. Ann Rheum Dis 2016;75:1909-16.

12. Kalunian KC, Merrill JT, Maciuca R, et al. A Phase II study of the efficacy and safety of rontalizumab (rhuMAb interferon- $\alpha$ ) in patients with systemic lupus erythematosus (ROSE). Ann Rheum Dis 2016;75:196-202.

13. Lazaro Cebas A, Cortijo Cascajares S, Pablos Bravo S, et al. Subcutaneous versus intravenous administration of trastuzumab: preference of HER2+ breast cancer patients and financial impact of its use. J BUON 2017:22:334-9.

14. Pivot X, Gligorov J, Müller V, et al. Patients' preferences for subcutaneous trastuzumab versus conventional intravenous infusion for the adjuvant treatment of HER2-positive early breast cancer: final analysis of 488 patients in the international, randomized, two-cohort PrefHer study. Ann Oncol 2014;25:1979-87.

15. Sylwestrzak G, Liu J, Stephenson JJ, et al. Considering patient preferences when selecting anti-tumor necrosis factor therapeutic options. Am Health Drug Benefits 2014;7:71-81.

16. Barbee MS, Harvey RD, Lonial S, et al. Subcutaneous versus intravenous bortezomib: efficiency practice variables and patient preferences. Ann Pharmacother 2013;47:1136-42.

17. Stoner KL, Harder H, Fallowfield LJ, et al. Intravenous versus subcutaneous drug administration. Which do patients prefer? A systematic review. Patient 2014

18. Pivot X, Gligorov J, Müller V, et al. Preference for subcutaneous or intravenous administration of trastuzumab in patients with HER2positive early breast cancer (PrefHer): an open-label randomised study. Lancet Oncol 2013;14:962-70.

19. Goldberg A, Geppert T, Schiopu E, et al. Dose-escalation of human anti-interferon- $\alpha$ receptor monoclonal antibody MEDI- 546 in subjects with systemic sclerosis: a phase 1, multicenter, open label study. Arthritis Res Ther 2014;16:R57.

20. Narain S, Furie R. Update on clinical trials in systemic lupus erythematosus. Curr Opin Rheumatol 2016;28:477-87.

21. Huynh TK, Ostergaard A, Egsmose C, et al. Preferences of patients and health professionals for route and frequency of administration of biologic agents in the treatment of rheumatoid arthritis. Patient Prefer Adherence 2014;8:93-9.

22. Jin JF, Zhu LL, Chen M, et al. The optimal choice of medication administration route regarding intravenous, intramuscular, and subcutaneous injection. Patient Prefer Adherence 2015;9:923-42.

23. Keizer RJ, Huitema AD, Schellens JH, et al. Clinical pharmacokinetics of therapeutic monoclonal antibodies. Clin Pharmacokinet 2010;49:493-507.

24. Richter WF, Bhansali SG, Morris ME. Mechanistic determinants of biotherapeutics absorption following $\mathrm{SC}$ administration. AAPS $J$ 2012;14:559-70.

25. Richter WF, Jacobsen B. Subcutaneous absorption of biotherapeutics: knowns and unknowns. Drug Metab Dispos 2014;42:1881-9.

26. Ronnblom L. The importance of the type I interferon system in autoimmunity. Clin Exp Rheumatol 2016;34(Suppl 98):21-4.

27. Browhawn P, Santiago L, Morehouse C, et al. Target modulation of a type I interferon gene signature and pharmacokinetics of anifrolumab in a phase Ilb study of patients with moderate to severe systemic lupus erythematosus. Arthritis Rheumatol 2015;67(Suppl 10):2215.

28. Dickson MC, Ludbrook VJ, Perry HC, et al. A model of skin inflammation in humans leads to a rapid and reproducible increase in the interferon response signature: a potential translational model for drug development. Inflamm Res 2015;64:171-83. 